\title{
Optimal Unemployment Insurance and Employment History
}

\author{
Hugo A. Hopenhayn \\ Universidad Torcuato Di Tella and University of Rochester \\ Juan Pablo Nicolini* ${ }^{\dagger}$ \\ Universidad Torcuato Di Tella
}

September 29, 2005

\begin{abstract}
This paper considers the optimal design of unemployment insurance contracts in an environemnt in which workers experience multiple unemployment spells. The environment is suited to study the optimality of employment history related restrictions typically found in existing unemployment insurance programs. We show that when the principal cannot distinguish quits from layoffs, optimality calls for contracts with employment history contingent transfers. In particular, we show that the employment tax an employed worker pays decreases and the unemployment benefit he is entitled to in case of unemployment increases with job duration in the optimal contract. We show that these properties hold both when workers have incentives to quit form good jobs and when they have incentives to take bad jobs.
\end{abstract}

Keywords: Unemployment insurance, optimal contract. JEL: I3, J2, J6.

*Corresponding author. Miñones 2159, Capital Federal, (C1428ATG), Argentina. email: juanpa@utdt.edu.

${ }^{\dagger}$ We thank Fernando Alvarez, Hal Cole, Narayana Kocherlacota, Victor Rios-Rull, two anonymous referees and seminar participants at CEMFI and Federal Resrve Bank of Minneapolis for suggestions. All remaining erros are ours. 


\section{Introduction}

Current unemployment insurance (UI) programs restrict coverage based on the employment record of unemployed workers. For instance, in the United States, a minimum of six months of employment is needed to qualify for benefits, and coverage ratios increase with the length of previous jobs. In addition, some of these restrictions have a significant impact on equilibrium outcomes. Indeed, there is evidence that in $\mathrm{Canada}^{1}$, coincidental with the minimum requirement number of periods - 12 months - to qualify for unemployment benefits, job termination rates double. The optimality properties of these parameters of UI systems have been neglected in theoretical work on optimal design, which has focused on the simplified case of a single unemployment spell ${ }^{2}$.This paper extends previous theoretical research, by considering the problem of optimal unemployment insurance design in a model of multiple unemployment spells.

We model unemployment insurance design as a repeated moral hazard problem. In our model, the search effort of unemployed workers cannot be perfectly monitored by the enforcement agency. As a consequence, the insurance mechanism must trade-off incentives for job search with unemployment duration risk. The unemployment insurance program specifies contingent transfers from the enforcement agency to the worker as a function of current and past employment/unemployment records. The optimal program is the one that minimizes the budget for a given ex-ante expected utility for the worker.

As a first exploration, in Section 2, we assume that termination rates are exogenous. We show that previous results for the case of a single unemployment spell have their analogues in our multiple spells case: transfers to unemployed workers decrease with the length of their unemployment spell, and reemployment taxes also increase with the length of the previous spells. Furthermore, these transfer schedules also decrease with previous unemployment spells. The intuition for these results is simple. Since agents are risk averse and value consumption smoothing, optimal incentives are provided by using permanent and not temporary reductions in consumption. Hence, the longer a worker is unemployed, the lower is his permanent consumption level.

\footnotetext{
${ }^{1}$ See Christofides and McKenna (1996) and Baker and Rea (1998).

${ }^{2}$ See Baily (1978), Shavel and Weiss (1979) and Hopenhayn and Nicolini (1997). Exceptions that study on-the-job moral hazard problems are Wang and Williamson (1996), (2002) and Zhao (2000).
} 
As job termination is exogenous, workers are insured completely against job loss. This property has two interesting implications. First, the optimal contract does not condition the transfers on the length of previous employment spells, so this environment does not provide a rationale to the employment history restrictions that motivates our analysis. Second, if an unemployed worker becomes employed and loses immediately this job, his replacement ratio is increased. This suggests the possibility of a loophole in the optimal contract, that can lead to opportunistic behavior. Indeed, anecdotal evidence in countries with generous unemployment insurance programs, suggests that, as job termination is a way to upgrade benefits, the UI program may induce inefficient quits. Obviously, this opportunistic behavior can only arise if the principal cannot distinguish quits from layoffs.

In the remaining section, the paper studies the design problem when quits and layoffs cannot be perfectly monitored. We explore two ways in which opportunistic workers may take advantage of the UI contract in this modified scenario.

First, we consider the case where the disutility of working and the generosity of unemployment insurance induce voluntary quits from socially efficient jobs. If the "no quit " constraint is not taken into account in the UI design, workers will look for jobs and once unemployed quit just to upgrade their benefits. When this constraint binds, it is optimal to condition the transfers on the agent's employment history. In particular we show that the tax workers pay while employed decreases with tenure while unemployment benefits rise. These properties hold for the entire length of the employment spell.

Second, we consider the case of job heterogeneity, where an added problem arises: unemployed workers may take bad jobs -that they would never accept in the absence of an unemployment insurance program- with the purpose of soon after quitting and requalifying for higher unemployment benefits. If the quality of jobs accepted by workers cannot be perfectly monitored, such behavior can arise introducing an added adverse selection problem. The optimal unemployment insurance design in the presence of such adverse selection problem shares most of the qualitative properties of the contract in the previous case, since employment taxes decrease and future unemployment entitlements rise with employment tenure. Yet there is one qualitative difference between the two cases: while in the first scenario the monotonicity properties hold for the entire employment spell, in the latter case they hold only for a finite period of time. From there on, both the employment tax and the future entitlement become constant. The explanation for this 
results is quite intuitive. The opportunistic strategy of taking a bad job and soon quitting to upgrade benefits is more costly the longer the worker remains in that job. As a consequence, the adverse selection constraint is relaxed with job tenure and ceases to bind after a finite number of periods. As this constraint is relaxed, workers with longer tenure can be provided better insurance against job loss.

The main contribution of the paper is to consider incentives and UI design in the presence of repeated unemployment spells. Three recent papers, Wang and Williamson (1996) and (2002) and Zhao (2000)) also derive an optimal contract that exhibits employment dependence. These papers differ from ours, in that they introduce a moral hazard problem for employed workers ${ }^{3}$. Even though the incentive problem we study - opportunistic quits - is of very different nature, their results are closely related to our first scenario, when the no quit constraint binds. The work of Wang and Williamson accounts for general equilibrium effects- and allows for private savings. However, given the complexity of their environment only simulation results are provided. While our environment abstracts from some of those features, we provide very general qualitative properties of the optimal contract. The paper by Zhao (which is contemporaneous to our paper) also provides a general characterization in a simple environment with unobservable job effort.

The paper proceeds as follows. In section 2, we describe the model with repeated unemployment spells in an environment where quits and layoffs can be distinguished by the principal. We relax this assumption in section 3 and study the optimal design when both, moral hazard and adverse selection incentive problems are present.

\section{The Model}

We model the unemployment insurance problem as a standard repeated moral hazard problem. Moral hazard arises as the principal (enforcement agency) cannot monitor an unemployed worker's search effort. The preferences of the agent are given by

$$
E \sum_{t=0}^{\infty} \beta^{t}\left[u\left(c_{t}\right)-a_{t}\right]
$$

\footnotetext{
${ }^{3}$ Wang and Williamson assume that on-the-job effort affects the probability to keep the job, while in Zhao it also affects the probability distribution of output.
} 
were $c_{t}$ and $a_{t}$ are consumption and search effort at time $t, \beta<1$ is the discount factor, common to the principal and agent, and $E$ is the expectations operator. The function $u$ is strictly increasing, strictly concave and unbouded above. For convenience, we assume that the effort level can take only two values, 0 or 1 . If effort is zero, the probability of finding a job is also zero, while if it is one, the probability of finding a job is some strictly positive number smaller than one called $p^{4}$.We also assume that all jobs are identical, offering a constant wage $w$ over time and terminating at a constant and exogenous rate $\lambda$.

We follow the literature in repeated moral hazard and assume that the principal can directly control the consumption of the agent or, equivalently, monitor its wealth. ${ }^{5}$ An unemployment insurance contract specifies, for each period, a net transfer to the agent and, if the agent is unemployed, a recommended action as a function of the realized history. Associated to each contract is an expected discounted utility to the agent $V$ and a cost to the principal $C$, measured by the expected discounted value of net transfers to the agent. These values assume that the agent responds to the contract rationally maximizing (1) by choosing the search effort. Given a level of lifetime utility for the agent at time zero, the optimal contract minimizes the cost of granting that utility to the agent in an incentive compatible way.

Incentive problems arise when the principal is interested in implementing positive search effort. In the following analysis we restrict to this case. The cost of implementing positive search effort increases with the promised utility to the agent. As a consequence, for sufficiently high initial utility levels, implementing high effort may not be optimal. In the appendix we solve the general problem and characterize the set of utilities such that the high effort is indeed optimal. The analysis in this section corresponds to utility levels within this set. ${ }^{6}$

\footnotetext{
${ }^{4}$ In Hopenhayn and Nicolini (1997) and in a preliminary version of this paper, we allowed for a continuum of effort levels. It significantly complicates the analysis without providing additional insights.

${ }^{5}$ According to Engen and Gruber (1995), the median 25-64 year old worker has gross financial assets equivalent to less than 3 weeks of income, and the average unemployment spell for those becoming unemployed is approimately 13.1 weeks. Gruber (1997) shows consumption falls by more than $20 \%$ for workers that enter unemployment with no claims to insurance.

${ }^{6}$ In the appendix we also show that for higher utility levels the solution is either trivial because there is no incentive problem or it is a lottery between the trivial solution and the one we solve for in this section.
} 


\subsection{Recursive contract}

Consider first the situation of an unemployed worker. In each period, the contract specifies current consumption $c$ and effort $a$, together with continuation values, contingent on the employment status of the agent at the end of the period. Let $V^{e}$ correspond to next period entitlement if the worker finds a job at the end of the current period, and $V^{u}$ the corresponding expected discounted utility if he does not find a job. Since $V$ is the expected discounted utility offered by the contract to the worker at the beginning of the current period, and we assumed $a=1$, then

$$
V=u(c)-1+\beta\left(p V^{e}+(1-p) V^{u}\right) .
$$

Given that search effort is not observed by the principal, the contract must satisfy the following incentive compatibility constraint:

$$
\beta p\left(V^{e}-V^{u}\right) \geq 1
$$

Let $C(V)$ denote the minimal budget necessary to provide a lifetime expected utility $V$ to an unemployed worker and $W(V)$ the corresponding bud-

get (possibly negative) for an employed worker. Then, the optimal problem when the worker is employed is given by

$$
\begin{aligned}
W(V) & =\min _{c, V^{e}, V^{u}} c-w+\beta\left[(1-\lambda) W\left(V^{e}\right)+\lambda C\left(V^{u}\right)\right] \\
\text { subject to } & : \quad u(c)+\beta\left((1-\lambda) V^{e}+\lambda V^{u}\right)=V
\end{aligned}
$$

With probability $\lambda$ employment terminates and the worker is granted continuation utility $V^{u}$ at a cost to the principal $C\left(V^{u}\right)$; with probability $(1-\lambda)$ employment continues, and the worker is granted continuation utility $V^{e}$ at a cost to the principal $W\left(V^{e}\right)$. The optimal choice of $c, V^{e}$ and $V^{u}$ minimize the cost of granting the value $V$ to the employed worker.

Consider now the dynamic programming equation when the worker is unemployed:

$$
\begin{aligned}
C(V) & =\min _{c, V^{e}, V^{u}} c+\beta\left(p W\left(V^{e}\right)+(1-p) C\left(V^{u}\right)\right) \\
\text { subject to } & : u(c)-1+\beta\left(p V^{e}+(1-p) V^{u}\right)=V \\
\text { and } & : \quad \beta p\left(V^{e}-V^{u}\right) \geq 1
\end{aligned}
$$


In the current period, the unemployed worker receives a transfer $c$. With probability $p$ the worker finds a job at the end of the period and is granted continuation utility $V^{e}$ at a cost to the principal $W\left(V^{e}\right)$; with probability $(1-p)$, the worker remains unemployed and is granted continuation utility $V^{u}$ at a cost to the principal $C\left(V^{u}\right)$. The optimal choices of $c, V^{e}$ and $V^{u}$ minimize the cost of granting this initial value $V$ to the unemployed worker subject to the incentive compatibility constraint.

\subsection{Characterization of the solution}

It is straightforward to verify that both functions $C(V)$ and $W(V)$ are increasing and strictly convex, as the corresponding return functions are linear, and the function $u$ in the constraints is strictly concave ${ }^{7}$. Consequently, these functions are almost everywhere differentiable.

$>$ From the first order and envelope conditions of the problem when the agent is employed, we obtain:

$$
W^{\prime}(V)=\frac{1}{u^{\prime}\left(c^{e}\right)}=W^{\prime}\left(V^{e}\right)=C^{\prime}\left(V^{u}\right) .
$$

By the strict convexity of $W$, it follows that $V=V^{e}$ so promised utility remains constant while employed. This has two important implications. Firstly, consumption of an employed worker is constant. If $c^{e}<w$, the worker will be taxed, otherwise he will get a subsidy. Second, and more importantly, the utility when the worker loses his job, $V^{u}$, is also independent of the length of the employment spell. Hence there is no employment dependence in the optimal unemployment insurance plan. This follows quite naturally from the fact that employment termination is exogenous. As there is no on-the-job moral hazard, the worker is completely insured against this shock.

Let us consider now the problem of the principal when the agent is unemployed. If we let $\delta$ be the multipliers of the incentive constraint, the first order and envelope conditions of this problem are

\footnotetext{
${ }^{7}$ A standard proof consists of replacing the control variable $c$ by its utility index $u=$ $u(c)$, which is a monotone transformation. With this transformation, all above constraints are linear. Since the inverse function $c(u)$ is convex, it follows that the return function for the principal is convex. Convexity of the value functions follows immediately from standard dynamic programming arguments.
} 


$$
\begin{aligned}
C^{\prime}(V) & =\frac{1}{u^{\prime}\left(c^{u}\right)} \\
C^{\prime}\left(V^{u}\right) & =C^{\prime}(V)-\delta \frac{p}{1-p} \\
W^{\prime}\left(V^{e}\right) & =C^{\prime}(V)+\delta
\end{aligned}
$$

It is straightforward to show that the incentive constraint always binds ${ }^{8}$. The next proposition characterizes the properties of the optimal contract.

Proposition 1 The unemployment benefit decreases and the reemployment tax increases with the length of the unemployment spell.

Proof. Note that the above first order conditions imply

$$
C^{\prime}(V)>C^{\prime}\left(V^{u}\right)
$$

and by the convexity of $C(\cdot)$ this implies that $V^{u}<V$, so the continuation utility decreases while the worker is unemployed. Moreover, the envelope condition

$$
C^{\prime}(V)=\frac{1}{u^{\prime}\left(c^{u}\right)} .
$$

implies that the benefit is increasing with $V$. Thus, over a single unemployment spell, the benefit decreases with the length of the spell. On the other hand, as the incentive compatibility constraint binds, the optimal continuation values $V^{u}$ and $V^{e}$ move in the same direction. Consequently, $V^{e}$ also decreases with the length of the unemployment spell. From the envelope condition of the problem while the worker is employed,

$$
W^{\prime}(V)=\frac{1}{u^{\prime}\left(c^{e}\right)} .
$$

As the wage is constant, this means that the tax is increases with the duration of the unemployment spell.

This proposition is a straightforward extension of Hopenhayn and Nicolini (1997) to the case of multiple unemployment spells: the use of higher

\footnotetext{
${ }^{8}$ Assume not, then, $V^{e}$ and $V^{u}$ are invariant over time, and $\frac{1}{u^{\prime}\left(c^{e}\right)}=W^{\prime}\left(V^{e}\right)=$ $C^{\prime}\left(V^{u}\right)=\frac{1}{u^{\prime}\left(c^{e}\right)}$ for all periods. Thus, the sequence of consumption is idependent of the employment state and unemployed workers have no incentive to choose $a=1$.
} 
reemployment taxes as part of the incentive structure follows from the efficiency of permanent income punishments when workers have a preference for consumption smoothing.

Corollary 1 All net future contingent transfers increase with the value of $V$.

Proof. This follows immediately from the last proposition.

The Corollary indicates the persistent effect of rewards and punishments. In particular, it implies that the replacement ratio and the reemployment tax both depend in a non-trivial way on all previous unemployment spells and on their duration. It should be noted that there is no simple statistic to compare different unemployment/employment histories. If two workers $a$ and $b$ experience the same number of unemployment spells but each spell is longer for worker $a$, this worker will face higher taxes or lower replacement ratios. But aside from this very strong ordering of unemployment histories, little else can be said. For example, take two workers $a$ and $b$ that start employed with identical $V$ (and thus the same consumption). Now suppose $b$ remains employed for the next two periods while $a$ loses the job after the first period and is reemployed after one period of unemployment. From equations (9-12) it follows that the final continuation utility of $a$ will exceed that of $b$, so its consumption will be higher (tax will be lower). ${ }^{9}$ This occurs, loosely, because while there is a reward for finding a job there is no punishment for losing it. (this can lead to opportunistic quits, as discussed in the next section). In particular, this implies that the total cumulative periods of unemployment or subsidies received in the past is not a sufficient statistic.

In providing incentives for search effort, the optimal contract generates a loophole, which could be exploited by workers to upgrade their unemployment benefits. In what follows, we discuss this issue.

\subsection{A loophole in the optimal contract}

The empirical literature on job duration provides some evidence that hazard rates for job termination tend to rise sharply at the time workers become eligible for unemployment insurance, suggesting the existence of opportunis-

\footnotetext{
${ }^{9}$ This fine point was indicated by a referee
} 
tic quits ${ }^{10}$. In this section we identify a loophole in the optimal insurance program derived above that leaves room for such opportunistic behavior.

Proposition 2 Consider an unemployed worker with promised future utility equal to $V$. If the worker finds a job and is fired the following period, the optimal contract will offer him a utility level $\overline{V^{u}}>V$.

Proof. Letting $V^{e}$ denote the promised utility when the worker finds the job, from the above first order conditions for the problems defining $C$ and $W$, it follows that

$$
C^{\prime}(V)<W^{\prime}\left(V^{e}\right)=C^{\prime}\left(\overline{V^{u}}\right) .
$$

By the convexity of the function $C$, it follows that $\bar{V}^{u}>V$.

This Proposition has two critical implications. Consider a worker that is unemployed. To provide incentives for searching, the worker must be better off getting a job than remaining unemployed. Thus, an immediate implication of the incentive constraint is that $V^{e}>V^{u}$. However, this does not imply that once employed, the worker is better off keeping the job rather than losing it. In particular, since $\bar{V}^{u}>V^{u}$ it could be the case that $\bar{V}^{u}>V^{e}$. This would give rise to opportunistic quit behavior. Moreover, getting a job, no matter how short-lived it is, upgrades unemployment benefits. This could give incentives for workers to fake employment, e.g. taking a bad job and then quitting, just to upgrade their unemployment insurance benefits. In either case, workers could abuse the UI system only if the principal is not able to monitor perfectly the causes of job termination. We analyze these cases in the following section.

\section{Quits and Layoffs}

In this section we assume that the principal cannot distinguish quits from layoffs. This is important, since UI is designed to protect workers against exogenous shocks. As quits may be considered, at least to some extent, endogenous, only fired workers should qualify for benefits. To cope with this

\footnotetext{
${ }^{10}$ For instance, in Canada job termination rates double at the 12 month period, which is the minimum period for eligibility (see Christofides and McKenna (1996) and Baker and Rea (1998)).
} 
problem, many unemployment insurance programs require involuntary separations to re-qualify for benefits. Admittedly, however, this distinction between involuntary and voluntary separations is hard to establish in practice, leaving room for opportunistic behavior. It is therefore natural to extend the model and study the optimal contract when the principal cannot distinguish quits form layoffs.

Two possible forms of opportunistic behavior may arise. First, if faced with very generous unemployment insurance, workers may quit their jobs to collect benefits and avoid the disutility of working. This case is considered in subsection 3.1. Secondly, when job offers are heterogeneous and the principal cannot monitor the quality of jobs, workers may take bad jobs and soon quit from them just to upgrade their unemployment benefits. This case is considered in subsection 3.2. As we show below, when any of these two cases occurs, it is optimal to condition benefits on employment duration. In particular, we show that in order to prevent either form of opportunistic behavior, replacement ratios rise with employment duration and the tax paid by an employed worker decreases with tenure.

\subsection{Quitting from good jobs}

So far we have assumed that effort at work does not affect the utility of an employed worker. For the analysis in the previous sections, this is just a normalization with no effect on any qualitative results. However, when quits and layoffs cannot be distinguished, workers could decide to quit opportunistically. As the following Proposition shows, the contract derived above is not immune to such behavior.

Proposition 3 Suppose $e \geq 0$ is the disutility of effort for an employed worker. Let $V^{e}$ and $V^{u}$ denote the utility value in the optimal contract, for a currently employed worker with initial utility $V^{e}$, who remains employed or loses the job, respectively.

1. If $e=0$, then $V^{e}>V^{u}$.

2. If $e>\frac{1}{p}$, then $V^{u}>V^{e}$

Proof. See appendix

This proposition implies that when the disutility of effort at work is sufficiently high, workers that find a job would certainly prefer quitting it after 
one period ${ }^{11}$ and restarting benefits at a higher level rather than continuing employed. To prevent this behavior, the optimal contract must guarantee that for an employed worker

$$
V^{e} \geq V^{u}
$$

where $V^{e}$ corresponds to the utility of keeping the job and $V^{u}$ the utility of losing it.

Adding this constraint, to the optimization problem defined by equation (4) and the promise-keeping constraint (5), we obtain a program that is almost identical to the one considered for an unemployed worker: if binding, the incentives needed to deter job quits are quite similar in nature to those needed to induce job search. Not surprisingly, the first order conditions for the two problems are almost identical. Consequently, if the no-quit constraint (13) binds,

$$
C^{\prime}\left(V^{u}\right)<W^{\prime}(V)<W^{\prime}\left(V^{e}\right)
$$

and if it does not bind

$$
C^{\prime}\left(V^{u}\right)=W^{\prime}(V)=W^{\prime}\left(V^{e}\right) .
$$

Together with the envelope conditions, the first equation implies that consumption falls when the worker loses the job (incomplete replacement) and it rises if the worker remains employed.

Let $\left\{V_{t}^{e}\right\}$ and $\left\{V_{t}^{u}\right\}$ denote the optimal continuation utilities derived from this program for a worker that starts employment at $t=0$ with an entitlement of utility $V_{0}^{e}$. The following Proposition shows that if the no-quit constraint binds in any period, then it must bind in all. It then follows from the above argument that the utility given by these two sequences is strictly increasing in $t$, the employment tenure. In particular, this implies that taxes while employed decrease with tenure and replacement ratios after becoming unemployed increase with the length of the previous employment spell.

Proposition 4 Suppose there exists an elapsed duration time $t$ such that the constraint $V_{t}^{e} \geq V_{t}^{u}$ binds. Then this constraint binds during all the employment spell. When this happens, the sequences $V_{t}^{e}$ and $V_{t}^{u}$ are strictly increasing.

\footnotetext{
${ }^{11}$ The sufficient condition $e>1 / p$ is far from necessary. Also note that no matter how large $e$ is, the incentive constraint of the unemployed problem ensures that workers that find a job are better off taking that job.
} 
Proof. See appendix.

Corollary 2 If the value of $e$ is high enough so that the no-quit constraint binds at the beginning of an employment spell, consumption will be strictly increasing (taxes decreasing) with tenure during this spell and the unemployment benefit the worker is entitled to increases with the length of the employment spell.

Proof. Follows immediately from the previous Proposition and the envelope conditions.

The intuition behind these results is the same as the one behind the falling replacement rates for an unemployed worker. The contract must make employment an attractive state and thus reward duration. Optimal rewards are permanent income rewards. Thus consumption in both, future employment and unemployment states must increase with tenure on the job. Finally note that when the incentive constraint binds, using equation (14) and the envelope conditions,

$$
\frac{1}{u^{\prime}\left(c_{t+1}^{u}\right)}=C^{\prime}\left(V_{t+1}^{u}\right)<W^{\prime}\left(V_{t}^{e}\right)=\frac{1}{u^{\prime}\left(c_{t}^{e}\right)}
$$

so consumption decreases when a worker becomes unemployed, i.e the replacement rate (after taxes) is less than one as occurs in most unemployment insurance programs.

\subsubsection{The optimal sequence of benefits}

Let us now turn to the problem of the principal when the worker is unemployed. Formally, the problem for an unemployed worker given by equations (6,7 and 8) remains unchanged, except for the definition of the function $W$, which is still increasing and convex. As far as the incentive constraint for search binds, the qualitative properties remain unchanged.

Proposition 5 The search incentive constraint binds.

Proof. Suppose the search IC constraint does not bind. Start with an unemployed worker with utility $V$ and initial consumption $c$. Since the IC constraint does not bind, if the worker continues unemployed his continuation 
utility and consumption will remain unchanged so $V \geq u(c)+\beta V$. In contrast, the value of getting a job is:

$$
\begin{aligned}
V_{e} & =u(c)-e+\beta V_{u} \\
& <u(c)-e+\beta V \\
& <V
\end{aligned}
$$

where consumption in the first period does not change by the contradiction hypothesis and the second line follow from the no quit constraint. But this immediately violates the search IC constraint.

As a consequence, with the exception of the employment dependence properties indicated above, all other qualitative characteristics of the unemployment insurance program ( i.e. that benefits decrease and taxes increase with the length of the current and previous unemployment spells) remain unchanged.

\subsection{Accepting bad jobs}

In this section we explore another potential source of incentive problems, namely, the possibility that workers may take bad matches and then quit from them, just to upgrade their unemployment benefits, exploiting the loophole indicated in section 2.3. In order to evaluate this possibility, we extend the model allowing for heterogenous job matches, the quality of which cannot be perfectly monitored by the principal. In contrast to current unemployment insurance generosity, which will typically lead workers to be excessively picky, future generosity may indeed have the opposite effect. ${ }^{12}$

A bad job offer, is here defined as one that is inefficient for an unemployed worker to take, and that in the absence of unemployment insurance the worker would never accept. The presence of such job opportunities introduces new constraints into the unemployment insurance design problem. In what follows, we characterize the unemployment insurance contract subject to these additional constraints, showing how employment dependence arises as an optimal response to prevent workers from exploiting these opportunities.

Bad jobs are socially inefficient because they provides a lower flow of utility. These jobs pay the same wage $w$ as the good jobs, but generate

\footnotetext{
${ }^{12}$ Mortensen (1977) considers this latter effect in a search model with multiple spells.
} 
disutility $d$ per period. A natural alternative would be to define the bad job as one with a lower wage $w_{0}$. In absence of unemployment insurance, the two problems are identical, letting $d=u(w)-u\left(w_{0}\right)$. However, this is not true for an unemployment insurance program that involves transfers during the employment state, since the marginal valuation of such transfers will differ depending on the wage received. Our separability assumption avoids this complication, and thus simplifies considerably the analysis ${ }^{13}$. In addition, for the analysis that follows, it is key that the principal cannot observe the quality of the job. We therefore find more natural to assume heterogeneity in utility which is unobservable. Given a search effort equal to one, bad jobs arise with positive probability and have constant termination rate $\lambda_{b} \in(0,1)$, which may be different from $\lambda$, the termination rate of the good jobs. As in the previous section we assume that quits and layoffs cannot be distinguished by the principal, for otherwise workers would never choose to take the bad job and the adverse selection constraint would never bind.

The bad job provides a costly way to send a signal of employment that the principal cannot distinguish from the signal corresponding to a good job. These alternative jobs are socially useless, in the sense that no worker would take them in the absence of unemployment insurance. However, an unemployment insurance contract with no employment dependence like the one considered before, may increase the private value of these jobs. We also assume, as it is standard in the search literature, that while employed, the worker cannot look for another job.

Instantaneous utility derived from the bad job is

$$
u(w)-d
$$

where $d$ is such that

$$
d>\alpha[u(w)-(u(0)-1)] .
$$

This inequality is imposed to make sure that in the absence of insurance, the worker is better off searching for the good job than taking the bad one. Indeed, if we let $V^{s}$ denote the value that an unemployed worker has -in the absence of any insurance- if it is optimal to search, it is easy to check that:

$$
(1-\beta) V^{s}=\alpha(u(0)-1)+(1-\alpha) u(w),
$$

\footnotetext{
${ }^{13}$ We conjecture that similar properties of the optimal unemployment insurance program would emerge without this separablity assumption. However, either strong conditions must be imposed in preferences to ensure that the problem is convex or randomizations might be optimal. We thank the comments of a referee that helped us to clarify this issue.
} 
where

$$
\alpha=\frac{1-\beta(1-\lambda)}{1-\beta(1-(\lambda+p))} .
$$

Equation (17) expresses the flow value of this unemployed worker as a weighted sum of the utility flow when unemployed $(u(0)-1)$ and the utility flow when employed $(u(w))$, where the weights $\alpha$ and $1-\alpha$ are derived from the rates of exit from unemployment $p$ and from employment $\lambda$, together with the discount factor.

On the other hand, the value of taking the bad job and quitting after one period $V_{b}$ is given by:

$$
V_{b}=u(w)-d+\beta V_{s} .
$$

The worker will not take the bad job, if and only if $V_{b} \leq V_{s}$, i.e

$$
u(w)-d<(1-\beta) V_{s}
$$

which after substituting for $V_{s}$ gives condition (16).

\subsubsection{The self-selection constraint}

Consider a given unemployment insurance contract, specifying net transfers to the worker $\tau_{t}$ as a function of the state (employed, unemployed) and the employment history $h$. Suppose this history is such that the worker is unemployed at time zero. Let $V$ indicate the value that the contract gives to the worker at this node. As in the previous section, let $V^{u}$ denote the value if the worker remains unemployed, $V^{e}$ the value if the worker gets a job and $c$ the current consumption (equal to the current period transfer $\tau$ ). The continuation contract for a worker that finds the job can be described by the two sequences: $\left\{c_{t}\right\}_{t=0}^{\infty},\left\{V_{t}^{u}\right\}_{t=1}^{\infty}$ which denote, respectively, the consumption in the first, second, third, etc. periods of employment and the continuation values if the job is terminated in the first, second, third, etc. periods of employment. Taking into account the constant probability of termination $\lambda$, it follows that

$$
V^{e}=\sum_{t=0}^{\infty} \beta^{t}(1-\lambda)^{t}\left[u\left(c_{t}\right)+\beta \lambda V_{t+1}^{u}\right] .
$$

In turn, the value of taking the bad job and quitting after $T$ periods is given by:

$$
Q_{T}=\sum_{t=0}^{T} \beta^{t}\left(1-\lambda_{b}\right)^{t}\left[u\left(c_{t}\right)-d\right]+\left(1-\lambda_{b}\right)^{T} \beta^{T+1} V_{T+1}^{u}
$$


In order for the worker not to take this alternative, it must be the case that for all $T \geq 0, V^{u} \geq Q_{T}$, i.e.

$$
V^{u} \geq \sum_{t=0}^{T} \beta^{t}\left(1-\lambda_{b}\right)^{t}\left[u\left(c_{t}\right)-d\right]+\left(1-\lambda_{b}\right)^{T} \beta^{T+1} V_{T+1}^{u},
$$

for all $T=0,1, \ldots$

Notice that for a given value entitlement $V^{e}$, these constraints restrict the particular paths $\left\{c_{t}, V_{t+1}^{u}\right\}$ than can be used to support that value. The optimal choice of this path determines the nature of employment dependence in the unemployment insurance contract. This problem is analyzed in the following section.

\subsubsection{Employment dependence: the optimal choice of $\left\{c_{t}, V_{t+1}^{u}\right\}$}

The optimal path $\left\{c_{t}, V_{t+1}^{u}\right\}$ is the solution to the following problem:

$$
W\left(V^{e}, V^{u}\right)=\min _{c_{t}, V_{t+1}^{u}} \sum_{t=0}^{\infty} \beta^{t}(1-\lambda)^{t}\left[c_{t}-w+\beta \lambda C\left(V_{t+1}^{u}\right)\right]
$$

subject to (18) and (19).

Before solving the problem, we must make sure that the choice set is well defined. Note that 18 imposes a lower bound on the sequence of controls, while 19 imposes upper bounds. Lemma 5 in the appendix establishes that this set is nonempty.

We now characterize the solution for the optimal path. Letting $\gamma$ and $\mu_{t}$ be the Lagrange multipliers of constraints 18 and 19 respectively and $\theta=\left(1-\lambda_{b}\right) /(1-\lambda)$ the first order conditions ${ }^{14}$ for the optimal (20) are:

$$
\frac{1}{u^{\prime}\left(c_{t}\right)}=\gamma-\theta^{t} \sum_{j=t}^{\infty} \mu_{j}
$$

and

$$
C^{\prime}\left(V_{t+1}^{u}\right)=\gamma-\theta^{t} \frac{\lambda_{b}}{\lambda} \sum_{j=t}^{\infty} \mu_{j}-\theta^{t} \frac{\left(1-\lambda_{b}\right)}{\lambda} \mu_{t}
$$

\footnotetext{
${ }^{14}$ The argument to prove that the value function is convex is the same as before, since the added constraint is linear in utilities.
} 
and the envelope conditions

$$
\begin{aligned}
W_{1}\left(V^{e}, V^{u}\right) & =\gamma \\
W_{2}\left(V^{e}, V^{u}\right) & =\sum_{j=0}^{\infty} \mu_{j}
\end{aligned}
$$

The interpretation of these partial derivatives is clear. First notice that if all multipliers $\mu_{t}$ are zero, the optimal path is constant, independent of the length of the employment spell. This corresponds precisely to the unconstrained problem discussed earlier. By the Kuhn-Tucker theorem, both $\gamma$ and the $\mu_{t}$ 's are positive, so until the self-selection constraint ceases to bind both $V_{t}^{u}$ and $c_{t}$ will be below their unconstrained values.

It is costly to hold a bad job and this cost increases with the length of employment. Since this cost is zero if the job is a good one, employment duration plays the role of a signal analogous to education signalling productivity in Spence (1974), where high productivity workers must invest enough in education to separate themselves. The larger is the cost for low productivity workers, the cheaper it is to separate the high productivity ones and less education is needed. In our model, the cost of staying on the bad job,

$$
\frac{d\left(1-\beta^{T+1}\right)}{(1-\beta)}
$$

is increasing with time. Thus, as time goes by, it becomes less costly to separate the two job types and the adverse selection problem. This suggests that that after some time period $T$ this cost of holding a bad job is sufficiently high that separating good from bad job becomes costless. The same intuition suggests that the profiles of consumption $c_{t}$ and $V_{t}^{u}$ should increase up to that period. This is indeed the case, as indicated in the following proposition.

Proposition 6 (i) There exists a $T$, such that the sequences $\left\{c_{t}\right\}$ and $\left\{V_{t+1}^{u}\right\}$ are strictly increasing up to that time period and constant thereafter. (ii) If the search IC constraint (3) binds, then $T<\infty$ and $\mu_{t}=0$ for all $t \geq T$.

Proof. See Appendix.

In order for the worker to have the right incentives, the optimal contract must punish early termination of jobs. As a result of the disutility of staying at the bad job $d$, the optimal contract can offer better insurance to the worker 
the longer he has been employed. Thus, the tax is a decreasing function of job length and the benefit received in case of unemployment is an increasing function. Eventually, for long enough duration, the incentive problems disappear and both the tax and future promised utility in case of unemployment become constant over time while employment lasts. Interestingly, the proof shows that the optimal sequence of benefits is independent of $\lambda_{b}$ on a set that includes $\lambda_{b} \in[\lambda, 1]$.

Replacement rates. Using (21) and (22) it follows that

$$
\begin{aligned}
C^{\prime}\left(V_{t+1}^{u}\right)-\frac{1}{u^{\prime}\left(c_{t}^{e}\right)} & =-\theta^{t} \frac{\lambda_{b}}{\lambda} \sum_{j=t}^{\infty} \mu_{j}-\theta^{t} \frac{\left(1-\lambda_{b}\right)}{\lambda} \mu_{t}+\theta^{t} \sum_{j=t}^{\infty} \mu_{j} \\
& =\frac{\theta^{t}}{\lambda}\left[\left(\lambda-\lambda_{b}\right) \sum_{j=t}^{\infty} \mu_{j}-\left(1-\lambda_{b}\right) \mu_{t}\right] .
\end{aligned}
$$

For $\lambda_{b} \geq \lambda$, the terms in bracket is strictly negative until the self-selection constraint stops binding. By the envelope theorem $C^{\prime}\left(V_{t+1}^{u}\right)=1 / u^{\prime}\left(c_{t}^{u}\right)$ this implies that $c_{t}^{u}<c_{t}^{e}$ so consumption falls when becoming unemployed. This results extends to the case $\lambda \geq \lambda_{b}$ since as shown in the Appendix (Lemma 9 ) the optimal policy in that case is identical to the one obtained for $\lambda=\lambda_{b}$. So as in the case of opportunistic quits, the (after tax) replacement rates are less than one.

Note that there is no adverse incentive problem if $T=0$, which would occur if the flow disutility $d$ is large relative to the upgrade in UI benefits. In the working paper version we show that, provided a continuity condition holds, there is a nonempty set of parameters for which $T>0$ and accepting bad jobs is not optimal for the planner. The argument relies on shrinking the time period -and appropriately rescaling probabilities- in such a way that it becomes essentially costless for the agent to take the bad job and quit an instant after ${ }^{15}$.

\subsubsection{The optimal sequence of benefits}

Let us now turn to the problem of the principal when the worker is unemployed. The only change needed to the first order conditions given in section

\footnotetext{
${ }^{15}$ We thank the comment of one referee that made us think about this issue.
} 
2.2 is that equation (11) needs to be replaced by:

$$
\begin{aligned}
C^{\prime}\left(V^{u}\right) & =C^{\prime}(V)-\delta \frac{p}{1-p}+p W_{2}\left(V^{e}, V^{u}\right) \\
& =C^{\prime}(V)-\delta \frac{p}{1-p}+\frac{p}{1-p} \sum_{j=0}^{\infty} \mu_{j} .
\end{aligned}
$$

Using (10) it follows that

$$
C^{\prime}(V)=(1-p) C^{\prime}\left(V^{u}\right)+p W_{1}\left(V^{e}\right)+p \sum_{j=0}^{\infty} \mu_{j}
$$

Letting $c$ denote the current consumption level for an unemployed worker, $c_{u}$ consumption in the following period if unemployed and $c_{e}$ consumption if employed, equations (26) and (21) imply the familiar inverse euler equation:

$$
\frac{1}{u^{\prime}(c)}=(1-p) \frac{1}{u^{\prime}\left(c_{u}\right)}+p \frac{1}{u^{\prime}\left(c_{e}\right)},
$$

which holds for all previous cases too.

If $\delta=0$, equation (25) implies that $c_{u} \geq c$, while the sequence of consumption for an employed worker is bounded above by $c$. It follows that $V^{u} \geq V^{e}$ and the worker would choose not to search. Hence $\delta>0$ and the search incentive constraint binds.

As in the previous section, with the exception of the employment dependence properties indicated above, all other qualitative characteristics of the unemployment insurance program (i.e. that benefits decrease and taxes increase with the length of the current and previous unemployment spells) remain unchanged.

While qualitative properties are alike, the opportunistic quits and adverse selection problems considered above are likely to have a quantitative impact on the evolution of both the benefit and the reemployment tax over time. We conjecture that, as the function $W$ becomes steeper and the marginal cost of providing utility at employment increases, the initial replacement ratios are likely to be higher but decrease more rapidly. Furthermore, in a setup where there is a range of search effort choices, one may conjecture that the optimal choice of effort will decrease. A full quantitative evaluation of the impact of this adverse section problem is left for future research. 


\section{Final Remarks}

In this paper we derive an optimal unemployment insurance program as the solution to a repeated principal agent problem. This paper extends previous work allowing for multiple employment/unemployment spells. We show that replacement rates decrease as a function of current and previous unemployment spells. The taxes paid by employed workers are also increasing in all previous unemployment spells. When job termination is exogenous, the history of current and previous employment duration has no effect on replacement rates or taxes.

In practice, most unemployment insurance schemes condition replacement ratios on the duration of previous employment spells. This paper provides a theoretical foundation for such practice. When quits and layoffs cannot be perfectly distinguished by the enforcing agency, the above optimal contract can lead to opportunistic quit behavior. Moreover, workers may also have an incentive to accept bad matches and soon quit from them, simply to upgrade their unemployment insurance benefits. Preventing such behavior imposes further constraints into the design of the insurance contract. Once these additional constraints are introduced, the optimal program calls for incomplete replacement ratios, which increase with the length of previous unemployment spells and taxes that decrease with tenure on the job.

Incentives are provided to make the employment state more attractive. Hence, the worker's expected future utility decreases with the length of unemployment spells and increases with the length of employment spells. This explains the duration dependence of unemployment benefits and taxes. Moreover, a change of state from unemployment to employment is rewarded and from employment to unemployment penalized, accounting for the incomplete replacement rate policy. The additional sources of opportunistic quit behavior examined in this paper increase the cost of providing search incentives for unemployed workers. We conjecture that this is likely to result in higher initial replacement ratios for the unemployed but a steeper decline with the length of unemployment. 


\section{A Proofs}

\section{A.1 Convexity of the optimal program when $e \in\{0,1\}$}

The optimal contract problem when the worker is unemployed has a discrete choice variable - the effort level. The general problem, as we will show, is not convex, so the unconstrained optimum may involve the use of lotteries.

Let

$$
\begin{aligned}
C_{0}(V) & =\min _{c, V^{u}} c+\beta C\left(V^{u}\right) \\
\text { subject to } & : \quad u(c)+\beta V^{u}=V
\end{aligned}
$$

and

$$
\begin{array}{r}
\qquad \begin{aligned}
C_{1}(V)= & \min _{c, V^{u}, V^{e}} c+\beta\left\{p W\left(V^{e}\right)+(1-p) C\left(V^{u}\right)\right\} \\
\text { subject to }: & u(c)-1+\beta\left\{p V^{e}+(1-p) V^{u}\right\}=V \\
& p\left(V^{e}-V^{u}\right) \geq 1
\end{aligned} \\
C(V)=\min _{q, V_{0}, V_{1}}(1-q) C_{0}\left(V_{0}\right)+q C_{1}\left(V_{1}\right) \\
\text { subject to }:(1-q) V_{0}+q V_{1}=V
\end{array}
$$

where $C_{0}$ and $C_{1}$ are the cost of recommending the low and high effort level today respectively, while $C$ is the cost of a lottery between the high effort level an the low effort level with the corresponding promised utilities. We show in the next proposition that the problem so defined is convex, so no further randomizations can improve upon the optimal solution.

Lemma 1 The functions $C_{0}, C_{1}$ and $C$ are convex.

Proof. If $C$ is convex, it is immediate to verify that the problem defined by equation (27) defines convex functions $C_{0}$ and $C_{1}$. We now show that if these two functions are convex, then equation (28) defines a convex function $C$. Let $\left(q, V_{0}, V_{1}\right)$ be solutions for $V$ and $q^{\prime}, V_{0}^{\prime}, V_{1}^{\prime}$ be solutions for $V^{\prime}$. Let

$$
V^{\lambda}=\lambda V+(1-\lambda) V^{\prime}
$$


and for this starting value take as choice variables

$$
\begin{aligned}
q^{\lambda} & =\lambda q+(1-\lambda) q^{\prime}, \\
V_{0}^{\lambda} & =\frac{\lambda(1-q) V_{0}+(1-\lambda)\left(1-q^{\prime}\right) V_{0}^{\prime}}{1-q^{\lambda}} \\
V_{1}^{\lambda} & =\frac{\lambda q V_{1}+(1-\lambda) q^{\prime} V_{1}^{\prime}}{q^{\lambda}}
\end{aligned}
$$

Simple algebra shows that this is a feasible solution for $V^{\lambda}$. Therefore, by definition

$$
C\left(V^{\lambda}\right) \leq\left(1-q^{\lambda}\right) C_{0}\left(V_{0}^{\lambda}\right)+q^{\lambda} C_{1}\left(V_{1}^{\lambda}\right)
$$

Convexity of $C_{0}$ and $C_{1}$ imply that

$$
C_{0}\left(V_{0}^{\lambda}\right) \leq \frac{\lambda(1-q) C_{0}\left(V_{0}\right)+(1-\lambda)\left(1-q^{\prime}\right) C_{0}\left(V_{0}^{\prime}\right)}{\left(1-q^{\lambda}\right)}
$$

and

$$
C_{1}\left(V_{1}^{\lambda}\right) \leq \frac{\lambda q C_{1}\left(V_{1}\right)+(1-\lambda) q^{\prime} C_{1}\left(V_{1}^{\prime}\right)}{q^{\lambda}}
$$

It therefore follows that

$$
\left(1-q^{\lambda}\right) C_{0}\left(V_{0}^{\lambda}\right)+q^{\lambda} C_{1}\left(V_{1}^{\lambda}\right) \leq \lambda C(V)+(1-\lambda) C\left(V^{\prime}\right),
$$

which completes the proof.

We do now show that the derivative of the $C_{1}$ function is larger than the derivative of the $C_{0}$ function, such that they display the single crossing property.

Lemma 2 For any $V, C_{0}^{\prime}(V)<C_{1}^{\prime}(V)$.

Proof. $\quad>$ From the first order and envelope conditions of the $C_{0}$ and $C_{1}$ problem we obtain

$$
\begin{gathered}
C_{0}^{\prime}(V)=\frac{1}{u^{\prime}\left(c_{0}\right)}=C^{\prime}\left(V_{0}^{u}\right) \\
u\left(c_{0}\right)+\beta V_{0}^{u}=V \\
C_{1}^{\prime}(V)=\frac{1}{u^{\prime}\left(c_{1}\right)}>C^{\prime}\left(V_{1}^{u}\right) \\
u\left(c_{1}\right)+\beta V_{1}^{u}=V
\end{gathered}
$$


Now, assume that $c_{0}<c_{1}$. The result follows from the concavity of $u$. Now, assume that $c_{0} \geq c_{1}$. Then, 30 and 32 and the convexity of $C$ imply that

$$
C^{\prime}\left(V_{0}^{u}\right) \leq C^{\prime}\left(V_{1}^{u}\right)
$$

The result follows from 29 and 31.

We can now characterize the function $C(V)$. First, if either $C_{0}(V)$ or $C_{1}(V)$ is below the other for all the domain, then $C(V)$ coincides with that curve. If they cross, by the single crossing property, they cross only once. As we already showed, $C_{1}(V)$ has a larger slope, so $C_{1}(V)$ will intersect $C_{0}(V)$ from below. Therefore, as it can be seen from the first order condition, the $C(V)$ function will coincide with $C_{1}$ for low values of $V$, will be linear for intermediate values of $V$, which means that randomizations are optimal, and will coincide with $C_{0}$ for high values of $V$. The analysis of the text is relevant for low values of $V$.

\section{A.2 Proof of Proposition 3}

Consider an employed worker. Let $V_{u}$ denote the utility of quitting. Under the optimal contract described in section 2.2, this is is the utility obtained by quitting any period. Consider the strategy: "stay this period but quit the next". The utility obtained equals:

$$
u\left(c_{e}\right)-e+\beta V_{u} .
$$

In turn, the strategy of quitting immediately gives utility:

$$
V_{u}=u\left(c_{u}\right)+\beta\left[p V^{e}+(1-p) \tilde{V}_{u}\right]
$$

which, as once the worker is unemployed the incentive constraint binds, can be written as

$$
V_{u}=u\left(c_{u}\right)+\beta \tilde{V}_{u}
$$

Under the above optimal contract, $c_{e}=c_{u}$, so the net gain of delaying one period the quit decision is:

$$
-e+\beta\left(V_{u}-\tilde{V}_{u}\right) .
$$


If $e=0$, then noting that $V_{u} \geq \tilde{V}_{u}$ (with strict inequality if reemployment is desired) it follows that the agent has no loss (strict gain) by delaying the quitting decision. Applying this principle recursively, the worker is better off by not quitting.

To prove part 2, suppose by way of contradiction that $e>1 / p$ but the value of employment $V_{e} \geq V_{u}$. Let $\tilde{V}_{e}$ denote the utility that an unemployed worker with initial value $V_{u}$ gets if he finds a job. We first show that $\tilde{V}_{e} \geq V_{u}$. Let $\tilde{c}_{e}$ be the first period consumption of this employed worker. As proved in section 2.2 consumption rises if an unemployed worker gets a job, so $\tilde{c}_{e} \geq$ $c_{u}=c_{e}$. Since consumption is monotonic in value, this implies that $\tilde{V}_{e} \geq V_{e}$ which by the contradiction assumptions is no less than $V_{u}$. The incentive constraint for the unemployed worker implies that $\beta\left(\tilde{V}_{e}-\tilde{V}_{u}\right)=1 / p$ which together with the previous result implies that $\beta\left(V_{u}-\tilde{V}_{u}\right) \leq 1 / p<e$, so the strategy of quitting immediately dominates the strategy of waiting one more period. Applying this result recursively, prover that $V_{e}<V_{u}$.

\section{A.3 Proof of Proposition 4}

First note that if this constraint does not bind at time $T$, then from equation (15) it follows that $V_{T}^{e}=V_{T+1}^{e}$ and by iterative application $V_{t}^{e}=V_{T}^{e}$ for all $t \geq T$. Moreover, as $V_{t}^{e}$ remains constant so does $V_{t}^{u}$ and thus $V_{t}^{e} \geq V_{t}^{u}$ for all $t \geq T$ and the constraint does not bind for any future period. Next we show that if the constraint binds in some period $T$ then it must bind in all subsequent periods. The combination of these two results proves the first part of the proposition. Suppose, by way of contradiction, that (13) binds at $t$, i.e. $V_{t+1}^{u}=V_{t+1}^{e}$, but it does not bind at $t+1$. Using equations (14) and (15) it follows that:

$$
C^{\prime}\left(V_{t+1}^{u}\right)<W^{\prime}\left(V_{t}^{e}\right)<W^{\prime}\left(V_{t+1}^{e}\right)
$$

and

$$
C^{\prime}\left(V_{t+2}^{u}\right)=W^{\prime}\left(V_{t+1}^{e}\right)=W^{\prime}\left(V_{t+2}^{e}\right)
$$

By strict convexity of functions $C$ and $W$, and since $V_{t+1}^{u}=V_{t+1}^{e}$ it follows that

$$
V_{t+2}^{u}>V_{t+1}^{u}=V_{t+1}^{e}=V_{t+2}^{e},
$$

which violates the no-quit constraint.

That $V_{t}^{e}$ (and thus $V_{t}^{u}$ ) strictly increases when the no-quit constraint binds, follows immediately from equation (14). 


\section{A.4 Proofs for Section 3.2}

The proofs in this section follow from a series of intermediate Lemmas. The basic outline is as follows. Two cases are distinguished: $\lambda_{b} \leq \lambda$ and $\lambda_{b} \geq \lambda$. A key result that we establish for the first case is that the constrained optimal solution is the same for all $\lambda_{b}$ in that range, so we reduce the problem to proving all results for $\lambda_{b}=\lambda$. For the case $\lambda_{b}>\lambda$, the optimal program is not independent of $\lambda_{b}$ so a separate proof is given.

Lemma 3 Let $Q_{T}$ denote the value of taking the bad job at time zero and quitting at the end of period $T$. Then $Q_{T+1}-Q_{T}$ has the same sign as $u\left(c_{T+1}\right)-d+\beta V_{T+2}^{u}-V_{T+1}^{u}$.

Proof. Follows immediately by noting that:

$$
\begin{aligned}
Q_{T+1} & =\sum_{t=0}^{T+1} \beta^{t}\left(1-\lambda_{b}\right)^{t}\left(u\left(c_{t}\right)-d+\beta \lambda_{b} V_{t+1}^{u}\right)+\beta^{T+2}\left(1-\lambda_{b}\right)^{T+1} V_{T+2}^{u} \\
& =Q_{T}-\beta^{T+1}\left(1-\lambda_{b}\right)^{T+1} V_{T+1}^{u}+\beta^{T+1}\left(1-\lambda_{b}\right)^{T+1}\left(u\left(c_{T+1}\right)-d+\beta V_{T+2}^{u}\right) \\
& =Q_{T}+\beta^{T+1}\left(1-\lambda_{b}\right)^{T+1}\left(u\left(c_{T+1}\right)-d+\beta V_{T+2}^{u}-V_{T+1}^{u}\right) .
\end{aligned}
$$

Lemma 4 Take any $\lambda_{b}>0$. Consider a feasible plan $\left\{c_{t}, V_{t+1}^{u}\right\}$ such that the self selection constraint (19) binds until period $T \leq \infty$ and does not bind thereafter. Then that plan satisfies the self selection constraint for all other $\lambda_{b}$

Proof. The proof is by induction. First consider time $T=0$,

$$
u\left(c_{0}\right)-d+\beta V_{1}^{u}=V_{u}
$$

is the value of quitting for sure after one period and it is independent of $\lambda_{b}$, so it is obviously satisfied. Suppose the constraint holds for some $T_{0}<T$, so the value $Q_{T_{0}}$ of quitting at the end of period $T_{0}$ equals $V_{u}$. By Lemma 3, the sign of $Q_{T_{0}+1}-Q_{T_{0}}$ is independent of $\lambda_{b}$, and since by assumption it is zero for the given $\lambda_{b}$, it must be zero for all. Finally, we need to show that the constraint is satisfied for $T_{0}>T$. Again, we show this by induction. Period $T+1$ is the first period for which $\mu_{t}=0$. Hence from that point on $c_{t}=c$ 
and $V_{t+1}^{u}=V$ are constant. Since by assumption $Q_{T}=V_{u}>Q_{T+1}$ it follows that:

$$
u\left(c_{T+1}\right)-d+\beta V_{T+2}^{u}<V_{T+1}^{u}
$$

or equivalently,

$$
u(c)-d+\beta V<V_{T+1}^{u} .
$$

But this implies that $Q_{T+1}-Q_{T}<0$ for all $\lambda_{b}$, so the self-selection constraint is slack for $T+1$. Finally, note that since $V \geq V_{T+1}^{u}$, it follows from equation (33) that

$$
u(c)-d+\beta V<V,
$$

so $Q_{t}>Q_{t+1}$ for all $t \geq T+1$.

The following Lemma uses the previous result to establish the existence of a feasible plan.

Lemma 5 For all $V^{u}$ and $V^{e}=V^{u}+\frac{1}{p \beta}$ there exist paths $\left\{c_{t}, V_{t+1}^{u}\right\}$ satisfying constraints (3), (18) and (19). Moreover, the paths can be chosen so that constraint (3) binds.

Proof. Pick any sequence $\left\{c_{t}, V_{t}^{u}\right\}$ such that constraint (19) is satisfied with equality for all $T$. By lemma 4 the constraint is satisfied for all $\lambda_{b}$. In particular, for $\lambda_{b}=\lambda$ the worker is indifferent between quitting or not any period. It follows that

$$
V^{u}=\sum_{t=0}^{\infty} \beta^{t}(1-\lambda)^{t}\left[u\left(c_{t}\right)-d+\beta \lambda V_{t+1}^{u}\right]
$$

Let $\hat{V}_{e}$ denote the value of taking a good job that is associated to this path. Subtracting (34) from (18), it follows that:

$$
\hat{V}_{e}-V^{u}=\frac{d}{1-\beta(1-\lambda)} .
$$

Using equation (16) it follows that

$$
\hat{V}_{e}-V^{u}>\frac{u(w)-(u(0)-1)}{1-\beta(1-(\lambda+p))} .
$$

To evaluate the right hand side of this equation, note that for search to be at all valuable, it must be the case that $(1-\beta) V^{s}>u(0)$, where the second 
term is the value of never searching. In conjunction with equation (17) this implies that

$$
(u(w)-u(0))>\frac{\alpha}{(1-\alpha)}=\frac{1-\beta(1-\lambda)}{\beta p}
$$

which together with (35) implies that $\hat{V}_{e}-V^{u}>\frac{1}{\beta p}$. This path satisfies the incentive constraint. Moreover, it follows that by decreasing some components of the sequence an alternative path that satisfies constraint (19) and gives a value $V_{e}-V^{u}=\frac{1}{\beta p}$ can be found.

The remainder of this section proves that the optimal plan has strictly increasing sequences $\left\{c_{t}, V_{t+1}^{u}\right\}$ up to some $T$ and constant thereafter. The following two lemmas give intermediate results that are used in some of the proofs.

Lemma $6 V_{t+2}^{u}-V_{t+1}^{u}$ has the same sign as $\lambda_{b}\left(\lambda_{b}-\lambda\right) \sum_{j=t+1}^{\infty} \mu_{j}+(1-$ 入) $\mu_{t}-\left(1-\lambda_{b}\right)^{2} \mu_{t+1}$.

Proof. >From equation (22) it follows that

$C^{\prime}\left(V_{t+2}^{u}\right)-C^{\prime}\left(V_{t+1}^{u}\right)=\theta^{t} \frac{\lambda_{b}}{\lambda} \sum_{j=t}^{\infty} \mu_{j}+\theta^{t} \frac{\left(1-\lambda_{b}\right)}{\lambda} \mu_{t}-\theta^{t+1} \frac{\lambda_{b}}{\lambda} \sum_{j=t+1}^{\infty} \mu_{j}-\theta^{t+1} \frac{\left(1-\lambda_{b}\right)}{\lambda} \mu_{t+1}$

having the same sign as $\lambda_{b}(1-\theta) \sum_{j=t+1}^{\infty} \mu_{j}+\mu_{t}-\theta\left(1-\lambda_{b}\right) \mu_{t+1}$ which in turn has the same sign as $\lambda_{b}\left(\lambda_{b}-\lambda\right) \sum_{j=t+1}^{\infty} \mu_{j}+(1-\lambda) \mu_{t}-\left(1-\lambda_{b}\right)^{2} \mu_{t+1}$. The claim follows from the convexity of $C$.

Lemma 7 Assume $\lambda_{b} \geq \lambda$. Suppose $V_{t}^{u} \geq V_{t+1}^{u}$ and $Q_{t}=V_{u}$. Then $V_{t+2}^{u}<$ $V_{t+1}^{u}$ and $\mu_{t+1}>0$.

Proof. If $Q_{t}=V_{u}$, the incentive constraints imply that $Q_{t-1} \leq V_{u}=Q_{t}$, and $Q_{t}=V_{u} \geq Q_{t+1}$. These inequalities and Lemma 3 imply that

$u\left(c_{t}\right)-d+\beta V_{t+1}^{u} \geq V_{t}^{u} \geq V_{t+1}^{u} \geq u\left(c_{t+1}\right)-d+\beta V_{t+2}^{u}>u\left(c_{t}\right)-d+\beta V_{t+2}^{u}$,

(where $c_{t+1}>c_{t}$ follows from $(21)$ and $\theta \leq 1$ ) so $V_{t+1}^{u}>V_{t+2}^{u}$. Finally note that by Lemma 6 it follows that if $\mu_{t+1}=0$ and since $\lambda_{b} \geq \lambda$ then $V_{t+2}^{u}-V_{t+1}^{u} \geq 0$ contradicting the previous inequality. 
Lemma 8 In the optimal plan for $\lambda_{b} \geq \lambda$, the sequences $\left\{c_{t}, V_{t+1}^{u}\right\}$ are nondecreasing. Moreover, whenever $\mu_{t+s}>0$, for some $s \geq 0$, then $c_{t+1}>c_{t}$, and $V_{t+1}^{u}>V_{t}^{u}$. In addition, if $\mu_{t+s}=0$ for all $s \geq 0$, then $c_{t+s}=c_{t}$ and $V_{t+1+s}^{u}=V_{t+1}^{u}$ for all $s \geq 0$.

Proof. The results for $\left\{c_{t}\right\}$ follow immediately from (21) and $\theta \leq 1$. To show the results regarding $V_{t}^{u}$, consider first the case $\mu_{t}>0$ and suppose towards a contradiction that $V_{t}^{u} \geq V_{t+1}^{u}$. Applying repeatedly Lemma 7, $V_{t+s}^{u}>V_{t+s+1}^{u}$ and $\mu_{t+s}>0$ for all $s$. But since $\sum \mu_{s}<\infty$ and $\theta \leq 1$, $C^{\prime}\left(V_{t}^{u}\right) \rightarrow \gamma$ and so eventually it must increase. Now suppose instead that $\mu_{t}=0$. By the first order conditions (22) and since $\theta \leq 1$ it follows that $V_{t+1}^{u} \geq V_{t}^{u}$ and that $V_{t+1}^{u}=V_{t}^{u}$ only when $\mu_{t+s}=0$ for all $s \geq 0$.

The following Lemma proves that the optimal plan for $\lambda_{b} \leq \lambda$ is the same as that for $\lambda_{b}=\lambda$. Applying Lemma 8, it follows that the paths $\left\{c_{t}, V_{t+1}^{u}\right\}$ are nondecreasing for all $\lambda_{b}$.

Lemma 9 The optimal plan for $\lambda_{b} \leq \lambda$ is the same as that for $\lambda_{b}=\lambda$.

Proof. We first show that in the optimal plan for $\lambda_{b}=\lambda$ the self-selection constraint binds every period until some $T \leq \infty$ and ceases to bind thereafter. From Lemma 8, it follows that $V_{t+1}^{u} \geq V_{t}^{u}$. By Lemma 6 and using $\lambda_{b}=\lambda$, $V_{t+1}^{u}-V_{t}^{u}$ has the same sign as $(1-\lambda) \mu_{t-1}-\left(1-\lambda_{b}\right)^{2} \mu_{t}$ which implies that whenever $\mu_{t}>0, \mu_{t-1}$ must also be strictly positive. This implies that the plan for $\lambda_{b}=\lambda$ satisfies the assumptions of Lemma 4, so this plan is feasible for all $\lambda_{b}$. To show that indeed it is optimal for $\lambda_{b}<\lambda$ we construct multipliers $\left\{\tilde{\mu}_{t}\right\}_{t=0}^{T}$ that support this solution. Let $\theta=\left(1-\lambda_{b}\right) /(1-\lambda)$ and define

$$
\tilde{\mu}_{t}=\frac{1}{\theta^{t+1}}\left(\mu_{t}-(1-\theta) \sum_{j \geq t} \mu_{j}\right) .
$$

Using the first order conditions

$$
\begin{aligned}
\frac{1}{u^{\prime}\left(c_{t}\right)} & =\gamma-\theta^{t} \sum_{j=t}^{\infty} \tilde{\mu}_{j} \\
C^{\prime}\left(V_{t+1}^{u}\right) & =\gamma-\theta^{t} \frac{\lambda_{b}}{\lambda} \sum_{j=t}^{\infty} \tilde{\mu}_{j}-\theta^{t} \frac{\left(1-\lambda_{b}\right)}{\lambda} \tilde{\mu}_{t}
\end{aligned}
$$


by repeated substitution it follows that

$$
\begin{aligned}
\frac{1}{u^{\prime}\left(c_{t}\right)} & =\gamma-\sum_{j=t}^{\infty} \mu_{j} \\
C^{\prime}\left(V_{t+1}^{u}\right) & =\gamma-\sum_{j=t}^{\infty} \mu_{j}-\theta^{t} \frac{(1-\lambda)}{\lambda} \mu_{t} .
\end{aligned}
$$

The multipliers are positive and support the same allocation. This completes the proof.

Lemmas 8 and 9 prove part (i) of Proposition 6. The next Lemma is used to prove part (ii).

Lemma 10 Take a plan $\left\{c_{t}, V_{t+1}^{u}\right\}$ that satisfies the self selection constraints for some $\lambda_{b}$. Let $Q_{t}\left(\lambda_{b}\right)$ be the associated value of taking the bad job at time zero and quitting at the end of period $t$. Then if $Q_{t}\left(\lambda_{b}\right)=V^{u}$, then $Q_{t}\left(\tilde{\lambda}_{b}\right) \leq$ $V^{u}$ for $\tilde{\lambda}_{b} \geq \lambda_{b}$.

Proof. For all $t \leq T$ define

$$
U_{t}\left(\lambda_{b}\right)=u\left(c_{t}\right)-d+\beta \lambda_{b} V_{t+1}^{u}+\beta\left(1-\lambda_{b}\right) U_{t+1}\left(\lambda_{b}\right)
$$

where $U_{T+1}\left(\lambda_{b}\right)=V_{T+1}^{u}$. This is the value $-t$ periods after entry- of staying in the bad job and quitting for sure at $T+1$. We first prove inductively that

$$
Q_{T}\left(\lambda_{b}\right)-Q_{t}\left(\lambda_{b}\right)=\beta^{t+1}\left(1-\lambda_{b}\right)^{t+1}\left(U_{t+1}\left(\lambda_{b}\right)-V_{t+1}^{u}\right) .
$$

To simplify notation, we omit the argument $\lambda_{b}$ from $Q$ and $U$ functions. For $t=T-1$,

$$
\begin{aligned}
Q_{T}-Q_{T-1}= & \beta^{T}\left(1-\lambda_{b}\right)^{T}\left(u\left(c_{T}\right)-d+\beta \lambda_{b} V_{T+1}^{u}\right) \\
& +\beta^{T+1}\left(1-\lambda_{b}\right)^{T+1} V_{T+1}^{u}-\beta^{T}\left(1-\lambda_{b}\right)^{T} V_{T}^{u} \\
= & \beta^{T}\left(1-\lambda_{b}\right)^{T}\left\{\left[u\left(c_{T}\right)-d+\beta \lambda_{b} V_{T+1}^{u}\right]+\beta\left(1-\lambda_{b}\right) V_{T+1}^{u}-V_{T}^{u}\right\} \\
= & \beta^{T}\left(1-\lambda_{b}\right)^{T}\left(U_{T}\left(\lambda_{b}\right)-V_{T}^{u}\right) .
\end{aligned}
$$


To complete the induction, suppose $Q_{T}-Q_{t+1}=\beta^{t+2}\left(1-\lambda_{b}\right)^{t+2}\left(U_{t+2}-V_{t+2}^{u}\right)$.Then

$$
\begin{aligned}
Q_{T}-Q_{t}= & Q_{T}-Q_{t+1}+Q_{t+1}-Q_{t} \\
= & \beta^{t+2}\left(1-\lambda_{b}\right)^{t+2}\left(U_{t+2}-V_{t+2}^{u}\right)+\beta^{t+1}(1-\lambda)^{t+1}\left(u\left(c_{t+1}\right)-d+\beta \lambda_{b} V_{t+2}^{u}\right) \\
& +\beta^{t+2}\left(1-\lambda_{b}\right)^{t+2} V_{t+2}^{u}-\beta^{t+1}\left(1-\lambda_{b}\right)^{t+1} V_{t+1}^{u} \\
= & \beta^{t+1}\left(1-\lambda_{b}\right)^{t+1}\left[u\left(c_{t+1}\right)-d+\beta \lambda_{b} V_{t+2}^{u}+\beta\left(1-\lambda_{b}\right) U_{t+2}-V_{t+1}^{u}\right] \\
= & \beta^{t+1}\left(1-\lambda_{b}\right)^{t+1}\left(U_{t+1}-V_{t+1}^{u}\right) .
\end{aligned}
$$

An important implication of this result is that when $Q_{T}\left(\lambda_{b}\right)=V^{u}$, then $Q_{T}\left(\lambda_{b}\right) \geq Q_{t}\left(\lambda_{b}\right)$ for all $t$, so $U_{t+1}\left(\lambda_{b}\right) \geq V_{t+1}^{u}$. Moreover, we now show that when $Q_{T}\left(\lambda_{b}\right)=V^{u}, U_{t}\left(\lambda_{b}\right)$ is nonincreasing in $\lambda_{b}$ for all $t$. The proof is again by induction. For $t=T+1$ it is obviously satisfied, since $U_{T+1}=V_{T+1}^{u}$ is independent of $\lambda_{b}$. Now suppose it holds for $t+1$. Then

$$
U_{t}\left(\lambda_{b}\right)=u\left(c_{t}\right)-d+\beta \lambda_{b} V_{t+1}^{u}+\beta\left(1-\lambda_{b}\right) U_{t+1}\left(\lambda_{b}\right)
$$

and

$$
\frac{\partial U_{t}}{\partial \lambda_{b}}=\beta\left(V_{t+1}^{u}-U_{t+1}\left(\lambda_{b}\right)\right)+\beta\left(1-\lambda_{b}\right) \frac{\partial U_{t+1}}{\partial \lambda_{b}} .
$$

But as discussed above the first term is nonpositive and by the induction hypothesis so is the second term.

We are now ready to complete the proof. Fix $\lambda_{b}$ and suppose that the self-selection constraint binds for some $T$, i.e. $Q_{T}\left(\lambda_{b}\right)=V^{u}$. In particular, this implies that $Q_{T}\left(\lambda_{b}\right)-Q_{0}\left(\lambda_{b}\right)=\beta\left(U_{1}\left(\lambda_{b}\right)-V_{1}^{u}\right)$ is decreasing in $\lambda_{b}$. But $Q_{0}\left(\lambda_{b}\right)=u\left(c_{0}\right)-d+\beta V_{1}^{u}$ is independent of $\lambda_{b}$ and thus $Q_{T}\left(\lambda_{b}\right)$ is decreasing in $\lambda_{b}$.

We are now ready to prove part (ii) of Proposition 6 Take $\lambda_{b} \geq \lambda$. In every period $T$ where the self-selection constraint binds, $Q_{T}\left(\lambda_{b}\right)=V^{u}$, so by the previous lemma it follows that $Q_{T}(\lambda) \geq Q_{T}\left(\lambda_{b}\right) \geq V^{u}$. Note that

$$
\begin{aligned}
V^{u} & \leq Q_{T}(\lambda)=\sum_{t=0}^{T} \beta^{t}(1-\lambda)^{t}\left\{u\left(c_{t}\right)-d+\beta \lambda V_{t+1}^{u}\right\}+\beta^{T+1}(1-\lambda)^{T} V_{T+1}^{u} \\
& =\frac{-d\left(1-[\beta(1-\lambda)]^{T+1}\right)}{(1-\beta(1-\lambda))}+\sum_{t=0}^{T} \beta^{t}(1-\lambda)^{t}\left\{u\left(c_{t}\right)+\beta \lambda V_{t+1}^{u}\right\}+\beta^{T+1}(1-\lambda)^{T} V_{T+1}^{u} \\
& \leq \frac{-d\left(1-[\beta(1-\lambda)]^{T+1}\right)}{(1-\beta(1-\lambda))}+V^{e}
\end{aligned}
$$


This implies that

$$
V^{e}-V^{u} \geq \frac{d\left(1-[\beta(1-\lambda)]^{T+1}\right)}{(1-\beta(1-\lambda))}
$$

Now, assume towards a contradiction that for any $T \in \mathbb{R}, \exists s>0, \ni$ $\mu_{T+s}>0$. Then,

$$
V^{e}-V^{u} \geq \frac{d}{(1-\beta(1-\lambda))} .
$$

But as in the proof of lemma 5, this in turn implies that $V_{e}-V^{u}>\frac{1}{\beta p}$. As a consequence, the incentive compatibility constraint does not hold with equality, and $V_{e}$ can be reduced lowering total cost. This proves part (ii) of Proposition 6 for $\lambda_{b} \geq \lambda$. But by Lemma 9 the optimal plan for $\lambda_{b}<\lambda$ is exactly the same as the one for $\lambda_{b}=\lambda$. This completes the proof. 


\section{References}

[1] Anderson, P. and B, Meyer. "Unemployment insurance in the United States: layoff incentives and cross subsidies", Journal of Labor Economics, 1993.

[2] Baily, M. "Some Aspects of Optimal Unemployment Insurance " Journal of Public Economics, Vol. 10, 379-402, 1978.

[3] Baker, M. and S. Rea. "Employment spells and unemployment insurance eligibility requirements", The Review of Economics and Statistics, 8094, 1998.

[4] Christofides, L. and C. McKenna. "Unemployment insurance and job duration in Canada", Journal of Labor Economics, 286-313, 1996.

[5] Engen, Eric and J. Gruber, "Unemployment Insurance and Precautionary Savings," Journalof Monetary Economics, 47(3), June 2001, 545-579.

[6] Green, D. and C. Riddell. "The economic effects of unemployment insurance in Canada: An empirical analysis of UI disentitlement". Journal of Labor Economics, 1993.

[7] Gruber, J. "The Consumption Smoothing Benefits of Unemployment Insurance, "American Economic Review, vol. 87 no. 1, March 1997.

[8] Hopenhayn, H. and J.P. Nicolini. "Optimal Unemployment Insurance", Journal of Political Economy, Vol 105, 412-438, 1997.

[9] Hopenhayn, H. and J.P. Nicolini. "Employemnt rated Unemployment Insurance", Working Paper, Universidad Di Tella, 2002.

[10] Mortensen, D. "Unemployment Insurance and Job Search Decisions. " Industrial and Labor Relations Review, Vol. 4, July 1977.

[11] Phelan, C. and R. Townsend. "Computing multi-period information constrained optima." Review of Economic Studies, 1991.

[12] Shavell, S. and L. Weiss. "The optimal payment of unemployment insurance benefits over time." Journal of Political Economy, 1979. 
[13] Spear, E. and Srivastava, S., "On Repeated Moral Hazard With Discounting", Review of Economic Studies, 54, 599-617.

[14] Wang, C. and S. Williamson. "Unemployment Insurance with Moral Hazard in a Dynamic Economy", Carnegie-Rochester Conference Series in Public Policy, 44, 1-41, 1996.

[15] Wang, C. and S. Williamson. "Moral Hazard, Optimal Unemployment Insurance and Experience Rating", Journal of Monetary Economics , Vol. 49, pp. 1337-1371, 2002.

[16] Zhao, R. "The Optimal UI Contract: Why a Replacement Ratio?" mimeo, University of Chicago. 2000. 